\title{
Shielding effectiveness of original and modified building materials
}

\author{
T. Frenzel, J. Stumpf, and M. Koch \\ Leibniz University of Hanover, Institute of Electrical Engineering and Measurement Science, Germany
}

\begin{abstract}
This contribution deals with the determination of the shielding effectiveness of building materials used for office, factory and government buildings. Besides the examination of standard materials, measurements were also performed on modified materials, e.g. ferro concrete with enhanced shielding effectiveness due to a changed mixture or structure of the reinforcement. The measurements of original and modified materials were carried out in a fully anechoic room (FAR) according to IEEE 299-1997 from $80 \mathrm{MHz}$ up to $10 \mathrm{GHz}$.
\end{abstract}

\section{Introduction}

The shielding effectiveness (SE) of shielded rooms or buildings is of importance for the security of confidential information. It is administrable for the design of these rooms to take the SE of conventional building materials into account. By the acknowledgment the SE would be predictable and improvable in the design phase of further rooms or buildings. Currently investigations of building materials which are suited for the prevention of transmitted electromagnetic waves are not available for the frequency range from $80 \mathrm{MHz}$ up to $10 \mathrm{GHz}$. As a consequence the Leibniz University of Hanover (LUH) determines the SE of standard building materials and its slightly modified concepts in a survey by order of the German Federal Office for Information Security (BSI).

As aforementioned in this survey the determination of the shielding effectiveness of building materials and its modified concepts is relevant. These modifications are supposed to enhance the shielding in accordance to former theoretical conclusions. Also, these materials must be constructed in line with German building regulations. Therefore the expectations of the shielding effectiveness of the materials are in the range from 0 to $60 \mathrm{~dB}$.

To avoid inhomogeneity and varieties in measurement conditions of real buildings the buildings materials are

Correspondence to: T. Frenzel

(frenzel@geml.uni-hannover.de) constructed as separate walls with surface dimensions of $2 \mathrm{~m} \times 2 \mathrm{~m}$ and weights up to 3.5 tons. The measurements of these devices under test (DUT) are carried out in a fully anechoic room (FAR) and are performed on a specially designed measurement setup including test rack and anechoic chamber. In this paper, we present the design and validation of two DUTs representing inner walls made of plasterboards and two DUTs for exterior walls made of ferro concrete.

\section{Definition of the Shielding Effectiveness}

The SE describes the ability to prevent the transmission of electromagnetic waves from the outside to the inside or vice versa. In accordance to Gonschorek and Singer (1992), the shielding factor

$a_{S}$ is defined as the logarithmic ratio of the two power densities $S_{a}$ (power density without a shield) and $S_{i}$ (power density with a shield) in one fixed measurement point:

$a_{S}=10 \log _{10}\left(\frac{S_{a}}{S_{i}}\right)[\mathrm{dB}]$

Hence, the definition of the shielding factor using the electric and magnetic field strength is:

$a_{S}=20 \cdot 10 \log \left|\frac{E_{a}}{E_{i}}\right|[\mathrm{dB}]$

$a_{S}=20 \cdot 10 \log \left|\frac{H_{a}}{H_{i}}\right|[\mathrm{dB}]$

In the far field, the interdependence of the electric and magnetic field strength vector is based on the characteristic impedance of a plane wave in vacuum $Z_{0}=120 \pi \Omega$. Thereby, the shielding effectiveness based on radiated power $S E_{P}$ is obtained as:

$S E_{P}=10 \cdot 10 \log \left(\frac{P_{a}}{P_{i}}\right)[\mathrm{dB}]$ 


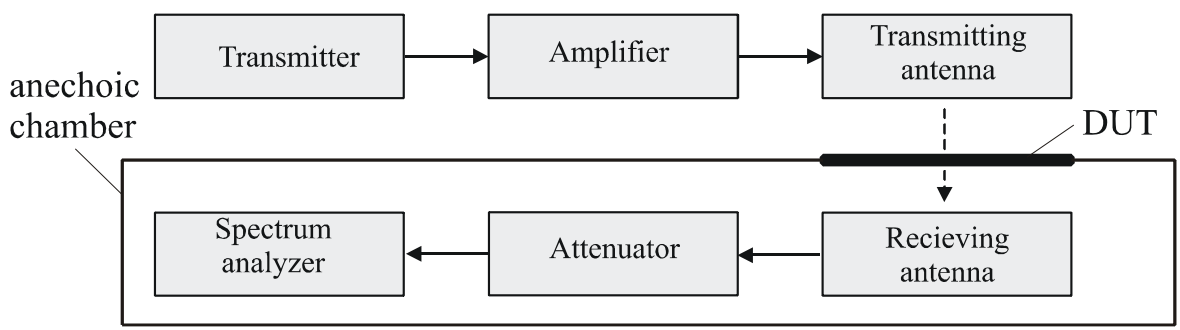

Fig. 1. Measurement setup according to IEEE 299-1997.

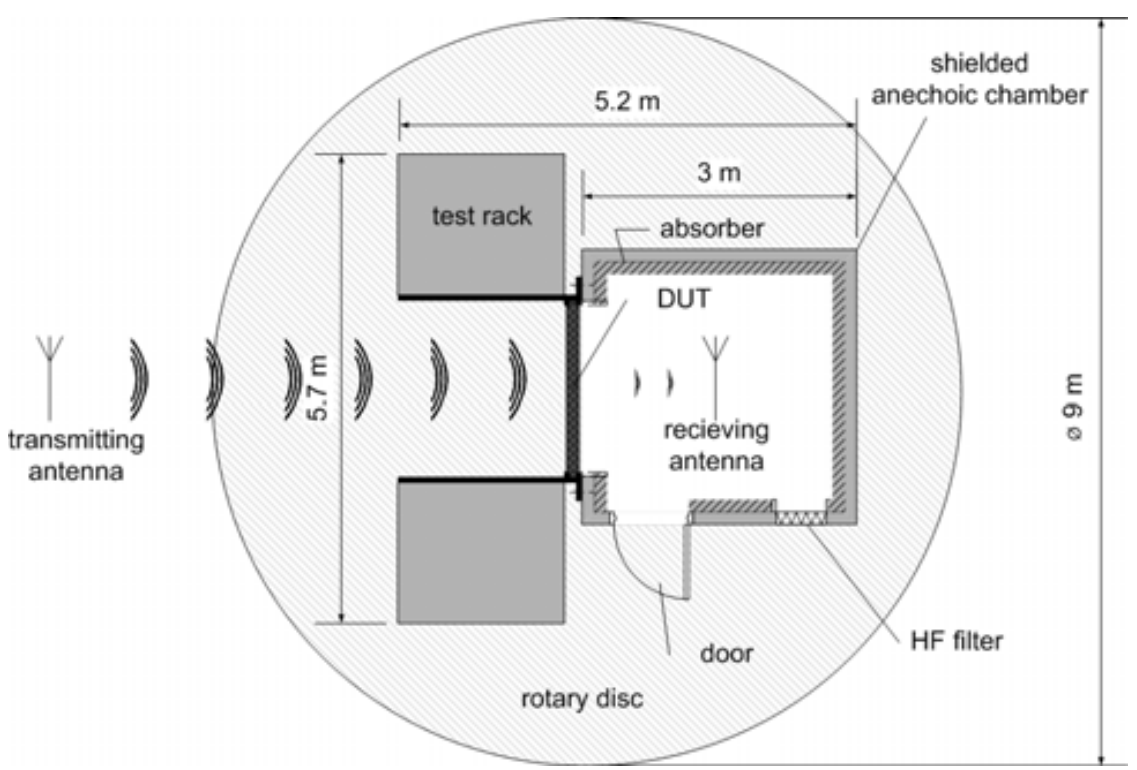

Fig. 2. Implemented measurement setup.

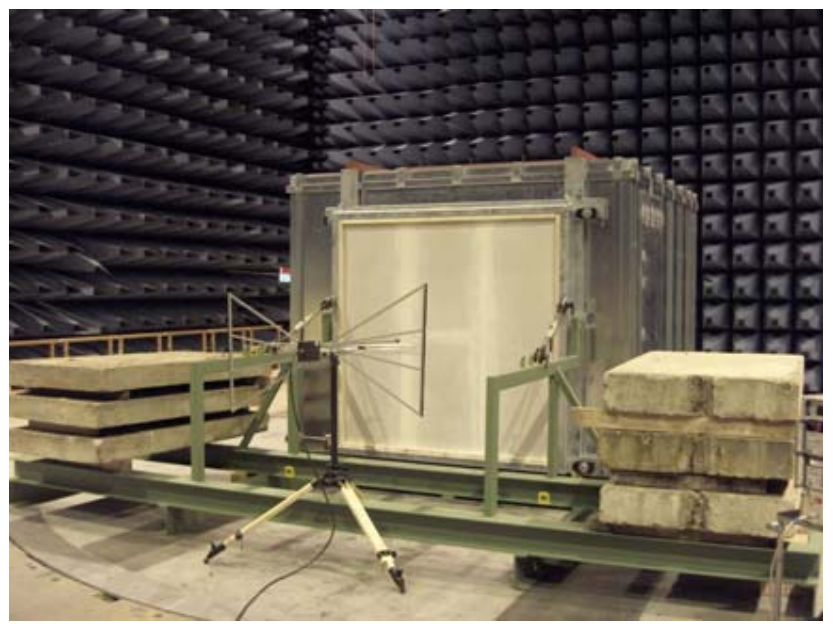

Fig. 3. Measurement setup in a fully anechoic room (WTD 81).

\section{Measurement setup}

The measurements are carried out in a fully anechoic room (FAR) according to IEEE 299-1997. The systematic setup is visualized in Fig. 1.

As it can be observed, the receiving unit is located inside the anechoic chamber, which is mounted to the DUT. Because of its heavy weight, the DUT is mounted on a specially designed test rack (see Fig. 2).

In accordance to IEEE 299-1997, the antennas shall be separated by certain distances, in order to maintain far field conditions. Thus, the receiving antenna inside the chamber is located at a distance of 0.8 meters at the centre of the DUT. As the FAR shown in Fig. 3, the anechoic chamber is fitted with absorbers to minimize resonance. On the backside of the chamber, where the highest level of incitation is expected, the wall is fitted with pyramidal absorbers with a height of $1.30 \mathrm{~m}$. Corresponding to Hemming (2002), they shall attenuate incident radiation with $f \geq 115 \mathrm{MHz}$ at a level of $30 \mathrm{~dB}$ or more. All other walls of the chamber are fitted with flat absorbers. 

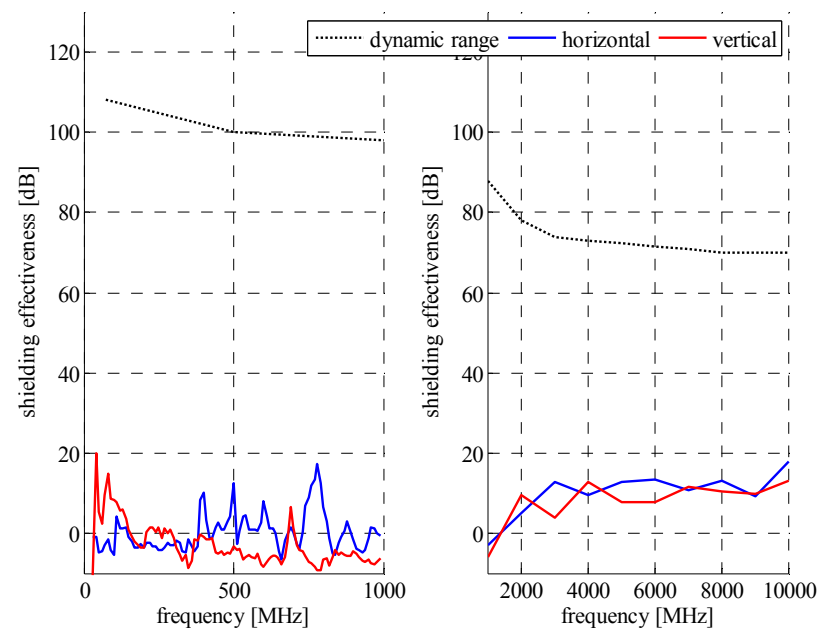

Fig. 4. Plasterboard with steel formwork and PE-foil.

According to Eq. (4), the shielding effectiveness of the four representative DUTs is determined by comparing the received field power with and without DUT in the frequency range from $80 \mathrm{MHz}$ to $10 \mathrm{GHz}$, splitted in two sections (0$1000 \mathrm{MHz}$ and $1-10 \mathrm{GHz}$ ) and validated separately. Measurements are performed with horizontal and vertical polarization.

The DUTs are designed according to German building regulations. They are constructed using a steel frame, which is mounted to the anechoic chamber. Conductive contact is arranged by finger stock gaskets and flexible copper mesh. Corresponding to German building regulations, the DUTs' thickness alternates in the range from 20 to $35 \mathrm{~cm}$.

The Plasterboard units consist of a steel formwork, with plasterboards mounted on the front and the back. In the hollow space between the two plasterboards, heat insulation is inserted, covered by a moisture barrier, which is made of polyethylene $(\mathrm{PE})$ foil or aluminium $(\mathrm{Al})$ foil.

The units of ferro concrete are made of standard concrete, both with enhanced reinforcement structure. Steel meshwork is doubled in a manner that results in a maximum mesh size of $7.5 \mathrm{~cm}$. For the significant difference, the first unit is additionally flush-mounted with expanded metal. The concrete of the second unit is not flush-mounted; instead it is compounded with steel-fibres in a size of $50 \times 1 \mathrm{~mm}$.

\section{Measurement results}

It is evident that the measurement setup affects the results of the measurement. Firstly, the cross-section of the DUT's frame (height $=1.9 \mathrm{~m}$, width $=1.9 \mathrm{~m}$ ) and secondly the crosssection of the anechoic chamber (height $=2.38 \mathrm{~m}$, width $=$ $3.0 \mathrm{~m}$, depth $=3.0 \mathrm{~m}$ ) influence the wave propagation. Ac-

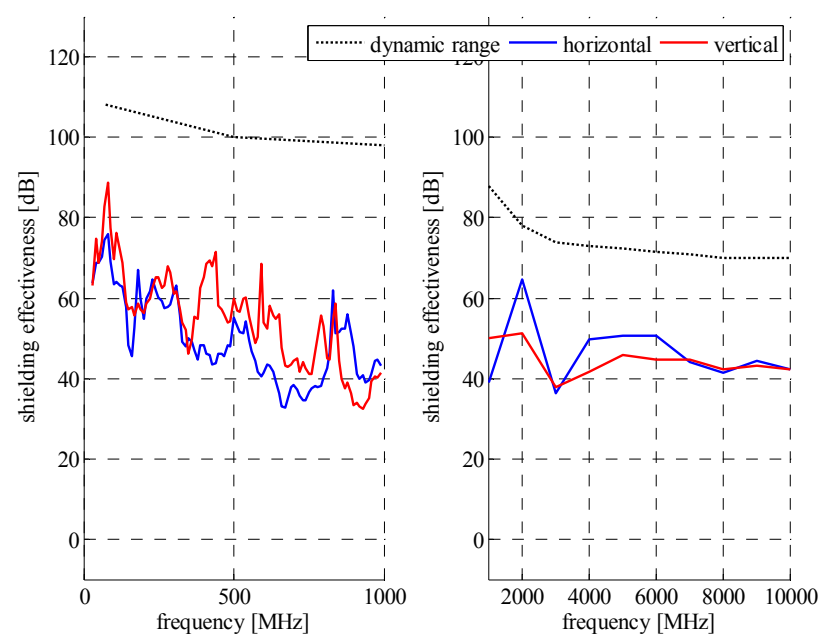

Fig. 5. Plasterboard with steel formwork and Al-foil.

cording to Meinke and Gundlach (1968) the following cutoff frequencies for $\mathrm{n}$ - and $\mathrm{m}$-modes are expected as:

$f_{\text {cut }}=\frac{\mathrm{c}}{2 \pi} \sqrt{k_{x}^{2}+k_{y}^{2}}=\frac{\mathrm{c}}{2 \pi} \sqrt{\left(\frac{m \pi}{\mathrm{h}}\right)^{2}+\left(\frac{n \pi}{\mathrm{w}}\right)^{2}}$

Below these cut-off frequencies a validation of the results due to the shielding effectiveness of the building materials is not permissible. Despite of fitting the anechoic chamber with absorbing material, resonance effects exist. These effects are minimized by comparing the incident field power as per IEEE 299-1997. Corresponding to Meinke and Gundlach (1968), the resonance frequencies for the lower n-, m-, and p-modes are estimated by:

$f_{\text {res }}=\frac{c_{0}}{2} \cdot \sqrt{\left(\frac{m}{d}\right)^{2}+\left(\frac{n}{h}\right)^{2}+\left(\frac{p}{w}\right)^{2}} ; m, n, p \in N$

In the following section the four representative building materials will be analysed, subdivided in the two frequency ranges, in consideration of these constraints.

\subsection{Plasterboard units}

Firstly, the measurement results of the plasterboard units are validated. As expected the shielding effectiveness of standard plasterboard with PE-foil is nearly zero (see Fig. 4), except for some resonances caused by the steel formwork. These resonances are indicated as peaks in the graph. For horizontal polarisation the steel formwork's horizontal reinforcement of $0.75 \mathrm{~m}$ represents a $\lambda$-dipole or a $2 \lambda$-dipole at the frequencies $R_{h, 1}=390 \mathrm{MHz}$ and $R_{h, 2}=780 \mathrm{MHz}$, respectively. This conclusion is applicable to the peak at $R_{v, 2}=690 \mathrm{MHz}$ for vertical polarisation, which is caused by the vertical reinforcement of $2.3 \mathrm{~m}$ length.

The SE for the $\mathrm{Al}$ foil is shown in Fig. 4. In contrast to the DUT with PE foil the SE is constantly higher than $35 \mathrm{~dB}$ 


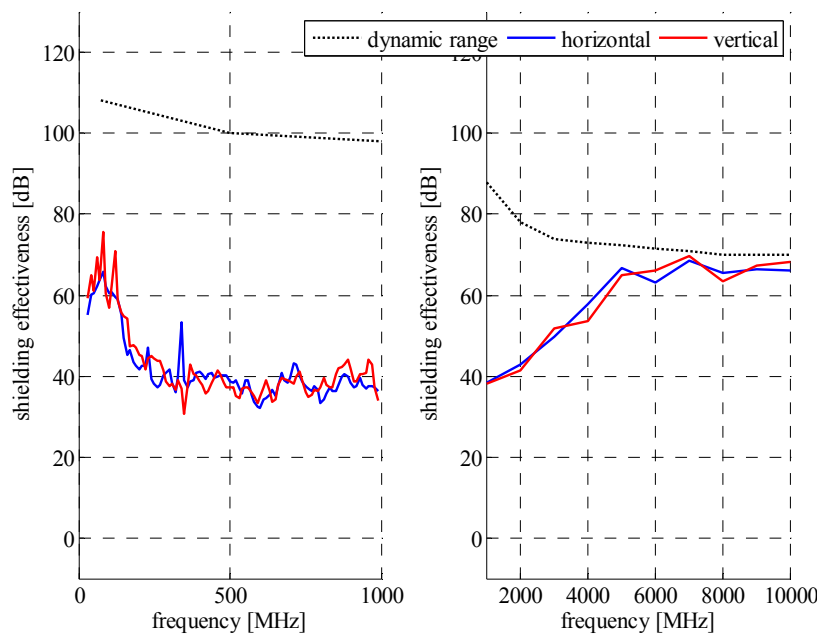

Fig. 6. Ferro concrete with expanded metal.

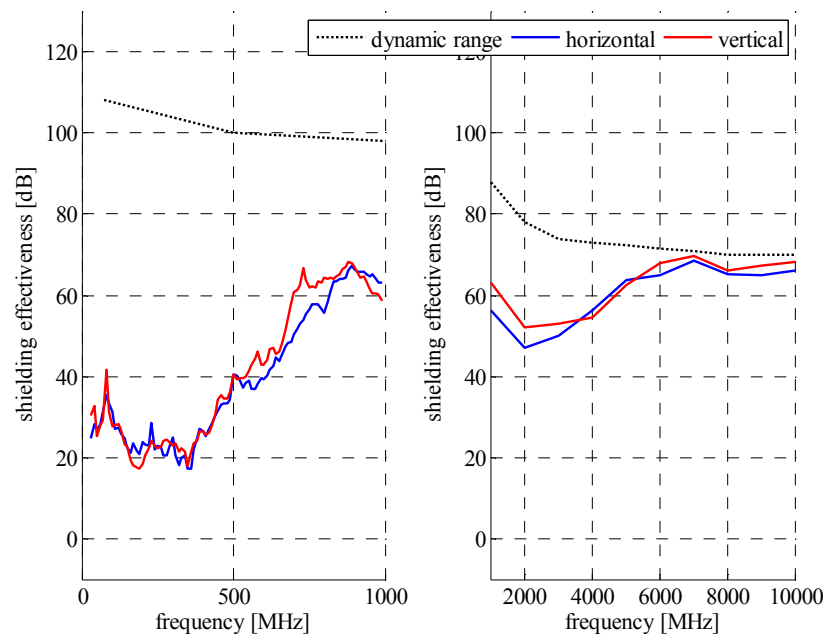

Fig. 7. Ferro concrete with steel fibres.

all over the frequency range. This effect is due to the aluminium foil. As the DUT with PE foil, the distribution of peaks caused by the horizontal and vertical reinforcements is of similar characteristic. Additional resonances are generated by not accurately taped seams of foil joints.

According to White (1980), the foil's thickness $t$ of $0.5 \mathrm{~mm}$ is much greater than the skin depth $\delta$ above $30 \mathrm{MHz}$ :

$\delta=\frac{1}{\sqrt{\pi f \mu_{r} \sigma_{r}}} \gg t$

With the frequency $f$, the relative permeability $\mu_{r}$ and the relative conductivity $\sigma_{r}$. Therefore it provides a better shielding effectiveness.
Table 1. Cut-off frequencies of frame and chamber.

\begin{tabular}{cccc}
\hline $\mathrm{m}$ & $\mathrm{n}$ & $\begin{array}{c}f_{\text {cut }} \\
(\mathrm{DUT} \text { 's frame) } \\
{[\mathrm{MHz}]}\end{array}$ & $\begin{array}{c}f_{\text {cut }} \\
\text { (anechoic chamber) } \\
{[\mathrm{MHz}]}\end{array}$ \\
\hline 1 & 0 & 78.89 & 49.97 \\
0 & 1 & 78.89 & 62.98 \\
\hline
\end{tabular}

Table 2. Lower resonance frequencies of the anechoic chamber.

\begin{tabular}{ccccc}
\hline $\mathrm{m}$ & $\mathrm{n}$ & $\mathrm{p}$ & $\begin{array}{c}\text { Resonance } \\
\text { frequencies } \\
{[\mathrm{MHz}]}\end{array}$ & $\begin{array}{c}\text { Wave length } \\
{[\mathrm{m}]}\end{array}$ \\
\hline 1 & 0 & 0 & 49.97 & 6.00 \\
0 & 1 & 0 & 49.97 & 6.00 \\
0 & 0 & 1 & 62.98 & 4.76 \\
1 & 1 & 0 & 70.66 & 4.24 \\
1 & 0 & 1 & 80.39 & 3.73 \\
0 & 1 & 1 & 80.39 & 3.73 \\
1 & 1 & 1 & 94.66 & 3.17 \\
0 & 2 & 0 & 99.93 & 3.00 \\
0 & 0 & 2 & 125.96 & 2.38 \\
2 & 2 & 0 & 141.32 & 2.12 \\
0 & 0 & 3 & 149.90 & 2.00 \\
\hline
\end{tabular}

\subsection{Ferro concrete units}

The major differences of the two ferro concrete DUTs are that the first one is covered by a layer of expanded metal (see Fig. 6), which the second is not. The concrete of the second DUT is compounded with steel fibres. For the unit with flush mounted expanded metal, the shielding effectiveness arises from the reinforcement of the flush (see Fig. 6). As a result of the small mesh size of the expanded metal, the cut-off frequency shifts up to smaller wave length. The high shielding effectiveness above $2 \mathrm{GHz}$ is due to the dipoles of incorporated water molecules in the concrete.

The curve of the DUT with steel fibres (Fig. 7) is similar for frequencies above $2 \mathrm{GHz}$ due to the water dipoles. In the frequency range from 80 to $350 \mathrm{MHz}$, the steel fibres do not affect the shielding effectiveness. Hence, by acknowledgement of the nearly non existing shielding of concrete the shielding is merely based on the enhanced reinforcement. In the range from 350 up to $1000 \mathrm{MHz}$, the rising shielding effectiveness is caused by the steel fibres, which are homogenously distributed in the concrete and solely incidentally connected. Thus, there is just a slight conductivity at low frequencies. But the conductivity is rising proportionally to the frequency according to capacitive coupling between fibres. Concerning Gonschorek and Singer (1992), the shielding factor $a_{S}$ of a shielded sphere rises due to the 
conductivity:

$a_{S} \approx 10 \log \left[\left(1+\frac{2}{3} \mu_{r} \frac{t}{r}\right)^{2}+\left(\frac{\omega \cdot \sigma \cdot r \cdot t}{3}\right)\right]$

With the frequency $\omega$, the relative permeability $\mu_{r}$, the relative conductivity $\sigma_{r}$, the sphere radius $r$ and the shield thickness $t$.

The results of the ferro concrete's shielding effectiveness differ from the plasterboard's. Thus the curve is not as much fluctuating and the effectiveness above $2 \mathrm{GHz}$ is much greater.

\section{Conclusions}

In this paper, four representative building materials were tested for shielding effectiveness. The measurements were carried out in a specially designed setup in a fully anechoic room. This setup is designed in accordance to IEEE 2991997. The devices under test are constructed as separate walls with dimensions of height $=2 \mathrm{~m}$, width $=2 \mathrm{~m}$, and weights up to 3.5 tons.

As expected, plasterboards and concrete are almost noneffective in shielding incident electromagnetic waves. But in the case of plasterboards the substitution of the conventional polyethylene moisture barrier with aluminium foil results in a significant rise of shielding effectiveness.

In the case of ferro concrete DUTs, enhanced reinforcement and expanded metal shifts the cut-off frequency up to higher frequencies, while steel fibres affect the shielding effectiveness from $350 \mathrm{MHz}$ up to $10 \mathrm{GHz}$. Further investigations shall validate these modifications in combination.

\section{References}

Gonschorek, K. H. and Singer, H.: Elektromagnetische Verträglichkeit: Grundlagen, Analysen, Maßnahmen, B. G. Teubner, Stuttgart, 1992.

Hemming, L. H.: Electromagnetic Anechoic Chambers, IEEE Press, New Jersey, 2002.

Meinke, H. and Gundlach, F. W.: Taschenbuch der Hochfrequenztechnik, 3 ed., Springer-Verlag, Berlin, Heidelberg, New York, 1968.

White, D. R. J.: A handbook on electromagnetic shielding materials and performance, 1980. 